\title{
Los mapas mentales como estrategia en el desarrollo de la inteligencia exitosa en estudiantes de secundaria
}

\section{Mental Maps as a Strategy in the Development of Successful Intelligence in High School Students}

\author{
Luis Alberto Núñez Lira* \\ Universidad César Vallejo, Lima, Perú \\ Universidad Nacional Mayor de San Marcos, Lima, Perú \\ ORCID: https://orcid.org/0000-0003-3542-9117 \\ Pedro Félix Novoa Castillo \\ Universidad César Vallejo, Lima, Perú \\ Universidad Nacional Mayor de San Marcos, Lima, Perú \\ ORCID: https://orcid.org/0000-0003-2186-7458 \\ Helga Ruth Majo Marrufo \\ Universidad César Vallejo, Lima, Perú \\ ORCID: https://orcid.org/0000-0002-5557-4269 \\ Angel Salvatierra Melgar \\ Universidad César Vallejo, Lima, Perú \\ Universidad Privada del Norte, Lima, Perú \\ ORCID: https://orcid.org/0000-0001-7318-0952
}

Recibido 11-09-18 Revisado 20-10-18 Aprobado 27-11-18 En línea 01-12-18

Correspondencia

Email: lnunezl@ucv.edu.pe

\section{Citar como:}

Núñez, L., Novoa, P., Majo, H., \& Salvatierra, A. (2019). Los mapas mentales como estrategia en el desarrollo de la inteligencia exitosa en estudiantes de secundaria. Propósitos y Representaciones, 7(1), 59-82. Doi: http://dx.doi.org/10.20511/pyr2019.v7n1.263

(c) Universidad San Ignacio de Loyola, Vicerrectorado de Investigación, 2019. 


\section{Resumen}

Los mapas mentales suelen ser empleados por docentes de todos los niveles educativos como potentes estrategias de aprendizaje. Empleo que permite logros pedagógicos importantes, pero que no llegan a traspasar su máximo nivel, por el uso dogmático del mapa mental originario de Tony Buzan (1996). Situación que sería distinta si se consiguiera internalizar, comprender y aplicarla en su cabalidad. Precisamente por ello, la teoría triárquica de la inteligencia desarrollada por Rober Sternberg (1985) permite a los estudiantes la capacidad de tomar decisiones ante diversas situaciones problemáticas con el uso adecuado de técnicas y orientaciones en el desarrollo mismo de la inteligencia. La investigación se desarrolló mediante el estudio de casos desde el enfoque cualitativo, donde a través del análisis de las evidencias encontradas y el empleo de un instrumento que midió la inteligencia exitosa, se pudo comprobar su desarrollo. Sin embargo, el análisis empírico no fue suficiente para validar dicho desarrollo; por ello se optó por la observación directa durante las sesiones de aprendizaje realizado por el docente responsable de la estrategia a emplear y plasmado en el cuaderno campo. Asimismo, las entrevistas a profundidad fueron llevadas a cabo por psicólogos educacionales. Cabe resaltar que los documentos analizados, han permitido realizar la triangulación no solo de evidencias sino también de metodologías, entre ellas el análisis documental, para llegar a conclusiones que demuestran de qué manera se ha logrado el desarrollo de la inteligencia exitosa. Por último, se demuestra que el uso de las técnicas de los mapas mentales con una estrategia educacional adaptada al grupo aplicado, logra el desarrollo significativo de las habilidades de la inteligencia analítica, creativa y práctica.

Palabras clave: Teoría triárquica; inteligencia analítica; inteligencia creativa; inteligencia práctica; mapas mentales.

\section{Summary}

Mind maps are usually employed by teachers from all the educational levels as powerful learning strategies. This use enables important pedagogic achievements, but they do not exceed their maximum level, due to the dogmatic use of Tony Buzan's mind map (1996). Situation that would be different if it were entirely internalized, understand and applied. Precisely for that reason, the triarchic theory of intelligence developed by Rober Sternberg (1985) allows students to make decisions in several difficult situations with the suitable use of techniques and orientations in the development of the same intelligence. The research was developed by means of the case study from the qualitative focus, in which through the analysis of the evidences found and the use of an instrument that measured the successful intelligence, its development could be confirmed. However, the empiric analysis was not enough for validating the aforementioned development. For that reason, direct observation was chosen during learning sessions accomplished by the teacher responsible for the strategy to be used, and embodied in the field notebook. Likewise, indepth interviews were conducted by educational psychologists. It should be noted that the analyzed documents have allowed developing the triangulation not only of evidences but also of methodologies, among them the documentary analysis, in order to reach conclusions that demonstrate how the development of the successful intelligence has been achieved. Finally, it is demonstrated than the use of the techniques of the mind maps with an educational strategy adapted to the applied group, achieves the significant development of the abilities of the analytical, creative and practical intelligence.

Keywords: Triarchic Theory; Analytical Intelligence; Creative Intelligence; Practical Intelligence, Mind Maps. 


\section{Introducción}

\section{Los mapas mentales.}

Dentro de los organizadores visuales que sistematizan la información con elementos análogos al procesamiento cerebral, destaca los mapas mentales, creados por Tony Buzan. Ya que este organizador de información se emparenta con el proceso de sinapsis (unión de dendritas) en su disposición de ramajes tanto en el trazo como en sus bifurcaciones. Según Molina y Martínez (2016) dentro de la vertiginosa trasformación que experimenta la sociedad, es imprescindible buscar nuevas formas de asegurar los logros educativos. Una de estas formas es el uso de los Mapas mentales, organizadores gráficos que representan y organizan de manera visual y estructural el conocimiento (p.11). Incluso, para González, Requena y Díaz (2015) esta técnica didáctica está muy entroncada con el modelo de aprendizaje tipo holístico; que, a su vez entiende el uso total del cerebro (p.402). La noción de holístico del pensamiento tiene relación con los tres niveles (Flavell, como se citó en González, Pareja \& Gea, 2016): metacognición de tareas o autoconciencia, metacognición de estrategias o autoconciencia de procedimientos y metacognición personal o autoconciencia cognitiva, donde la interconexión de todas ellas lleva a la autoconciencia del aprendizaje total (p.137).

Desde su aparición y sus diferentes análisis teóricos, se pueden establecer como elementos constitutivos principales al elemento central que dispara las asociaciones semánticas (pensamiento irradiante), así como las ramas que se van expandiéndose y dividiéndose (creando nódulos alternos) hacia afuera de acuerdo a la necesidad de estructuración del mapa. Su lógica de construcción se basa en la forma neuronal, por ello es que prefiere las líneas ondulantes a las rectas, por la idea de que el cerebro asimila mejor estas formas a las rígidas y geometrizadas de otros organizadores visuales de información. Es sustantivo señalar que el mapa mental incorpora tanto en su diseño como en su ejecución elementos lingüísticos (palabras, frases, oraciones) como no lingüísticos (codificación cromática, simbólica, etc.). Asimismo, dentro de la relación neuronal, los estudiosos han coincidido en señalar que estos organizadores activan tanto hemisferio izquierdo como el derecho, a diferencia de otros análogos que solo activan un solo hemisferio (solo el izquierdo). Desde la perspectiva de Ardila y Ostrosky-Solis (1991, p. 14) el cerebro humano dispone sus funciones de acuerdo a cada hemisferio así:

Tabla 1.

Funciones cerebrales por hemisferios.

\begin{tabular}{|c|c|}
\hline \multicolumn{2}{|c|}{ Organización funcional del cerebro } \\
\hline Hemisferio izquierdo & Hemisferio derecho \\
\hline $\begin{array}{l}\text { Codifica información sensorial con base en } \\
\text { descripción lingüística }\end{array}$ & $\begin{array}{l}\text { Codifica información sensorial en } \\
\text { términos de imágenes }\end{array}$ \\
\hline Análisis temporal & Sintetiza espacialmente \\
\hline $\begin{array}{l}\text { Efectúa comparaciones conceptuales } \\
\text { independientemente del contenido lingüístico }\end{array}$ & $\begin{array}{l}\text { Hace pareamiento visual sin realizar } \\
\text { comparaciones conceptuales }\end{array}$ \\
\hline Percibe detalles & Percibe forma \\
\hline Falta de un sintetizador gestáltico & Falta de analizador fonológico \\
\hline Comunicación verbal & $\begin{array}{l}\text { Maneja relaciones espaciales y efectúa el } \\
\text { análisis de las partes en relación con el } \\
\text { todo }\end{array}$ \\
\hline Procesamiento lingüístico y numérico & Reconocimiento perceptual de cosas \\
\hline Pensamiento analítico y secuencial & Pensamiento no verbal \\
\hline
\end{tabular}

Montero y De la Morena (2015, p.397) anota que los mapas mentales logran expresar de manera gráfica y no lineal una gran cantidad de información y datos sobre una temática determinada. Esto lo convierten en un instrumento ideal para aprovechar al máximo el potencial cerebral. Por otro lado, dentro la clasificación por la forma cómo se decodifica (lee e interpreta) 
son considerados textos discontinuos (que muchas veces son usados para la organización de información de textos continuos como lecturas convencionales de libros, artículos. Las imágenes que se forman en la mente (Perkins, como se citó en Martínez, 2017) permiten una categorización de conceptos, ordenados por criterios y esto constituye una contundente influencia creativa del tipo cognitivo. Asimismo, cada uno de las nociones conceptuales, cuyo empleo implica su operacionalización (Feldman, como se citó en Martínez, 2017) de objetos, hechos y toda clase de ideas que compartan características comunes entre sí. Actividad mental que le da orden y sistematiza los datos que le llegan del exterior, así como las que tiene en la memoria interna. La génesis de las imágenes mentales puede activarse por un ejercicio de evocación o imaginación (p.5).

Con la gran aceptación que tuvo el cognitivismo en los estudios pedagógicos, el propio Tony Buzán (como se citó en Blanco, 2017): asume la lógica de los mapas mentales como un método analítico que facilita a quien lo usa para sistematizar con mucha sencillez y certeza lo complejo del pensamiento, así como el aprovechamiento al máximo de la capacidad mental humana. Cualquiera de estos mapas son formas simples de emplear el discurrir de datos e información entre el cerebro y lo exterior. Constituyéndose, a su vez, como una herramienta eficaz y creativa en la nueva generación de más pensamiento e imaginación (p.26).

\section{Partes de un mapa mental.}

Los teóricos han coincidido en señalar los siguientes componentes básicos de un mapa mental, el presente estudio los vinculará a las nociones de la inteligencia triárquica entendida como habilidad analítica, práctica y creativa (Sternberg, 1985):

- IOB (Idea Ordenadora Básica): Es el componente principal del mapa mental, desde donde se van a expandir las asociaciones tanto semánticas como conceptuales del tema a organizarse y plasmarse en el mapa. Para Ontoria et al, 2006 la IOB va es el punto desde donde se irá jerarquizando las ideas secundarias. Enlazada con la inteligencia triárquica la IOB permite el punto de inicio analítico, así como su posterior bifurcación; logra potenciar la creatividad, ya que al usar un elemento icónico y cromático sintetiza o condensa semánticamente lo esencial de la información plasmada en el mapa; y por último, es práctica, porque confiere al diseño la jerarquización simple del centro hacia afuera.

- Ramificaciones: Es el entretejido estructural y subcategorial del mapa. A través de las ramas se establecen asociaciones y subdivisiones de acuerdo al diseño que se le imprima. Estas ramas se van irradiando del centro hacia afuera (Ontoria et al, 2006). Desde la lógica triárquica: permite especificar el análisis a niveles de detalle; configura lo práctico en poder subdividir las veces que se necesite para aclarar o explicitar mejor determinado concepto; y desde el punto creativo, se puede diseñar de manera muy libre el grosor, color y tono de las ramas, para añadirle énfasis en su trazado.

- Nódulos asociativos - Ideas Secundarias: Las ideas secundarias son una suerte de nódulos conceptuales que originarán a su vez ramas subdivisorias. Son pequeños focos de irradiación subordinados a la principal (IOB) que cumplen dentro de la inteligencia triárquica una finalidad analítica al ser puntos subcategoriales de división de una totalidad temática para su mejor comprensión; una finalidad creativa, porque le permite al sujeto colocar dentro de estos nódulos elementos cromáticos o icónicos que resuman o sinteticen alta carga semántica; y práctica, porque gracias a su ductibilidad, el diseño del mapa mental puede subdivirse en conceptos disgregados de manera jerárquica y secuencial. Los rótulos dentro de los nódulos asociativos deben estar expresados de manera clara y precisa (Buzan, 1996, p. 119).

- Codificación: El uso de una codificación simbólica, cromática o icónica es importante en el diseño y posterior empleo de cualquier mapa mental. Esta codificación permite asociarse con la inteligencia triárquica de manera práctica: al usar elementos culturales y comunes expresados en íconos, símbolo y significación cromática; de manera analítica, al emplearse dentro de los códigos, subdivisiones internas hasta los niveles que se 
requiera para el mejor entendimiento de los mapas mentales; y por último y el más importante, que expande de manera infinita la posibilidad creativa del pensamiento humano al plantear codificaciones iconográficas, pictóricas y alegorías de cualquier tipo y aprovecharlos en el mapa mental. Esta codificación suele concretizarse en el uso de esferas, elipsis, subrayados, soles, partes del cuerpo y otros gráficos simplificados que ahorren texto y redondeen ideas (Buzan, 1996). Así como también simbología numérica, flechas, formas geométricas y demás que contribuyan a jerarquizar, orientar o vincular nódulos en el mapa mental (Ontoria, 2006).

Por todo lo anterior, se puede hablar del uso de los mapas mentales para la Inteligencia exitosa en tanto se emplee este organizador como el ejercicio consciente y dirigido de las tres habilidades triárquica planteadas por Sternberg: habilidades analíticas, creativas y prácticas.

\section{La inteligencia.}

La inteligencia, cuyo concepto ha evolucionado a través de la historia vincula a la medición, desde Binet, que elaboró instrumentos cuyo objetivo fue la medición de los grandes procesos mentales, Binet y Simon en el año de 1905 que generan la escala de medición cuyos resultados indicaban a los niños que requerían una educación especial; Stern en 1911, desarrolla el concepto de coeficiente intelectual; Terman en 1916 desarrolla la escala Stanford-Binet desarrollando la escala de coeficiente intelectual; Spearman en 1904 desarrolló el "factor G de la inteligencia". En la figura 1 , se puede observar las correlaciones del factor $\mathrm{G}$ con los factores $\mathrm{S}$.

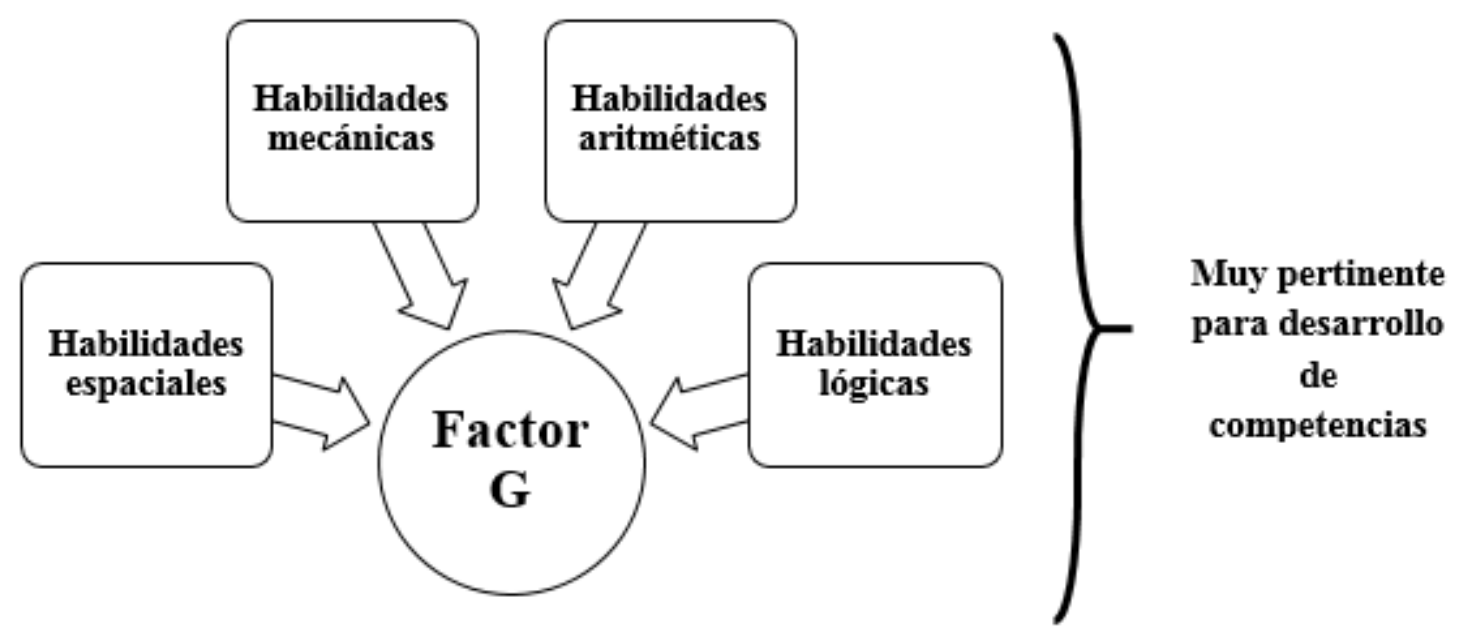

Figura 1. Modelo de Spearman

\section{Relación del factor $\mathbf{G}$ con los factores $S$.}

Aquí desarrolla las correlaciones entre los diversos factores; Vernon en 1964 desarrolla la inteligencia en función a las habilidades cognitivas, desde el factor $\mathrm{g}$ hasta los factores específicos. Catell en 1967 desarrolla la teoría de la inteligencia fluida y la inteligencia cristalizada, cuya finalidad fue la resolución de problemas. Considero los factores de "actores de percepción visual, memoria a corto plazo, almacenamiento y recuperación a largo plazo, velocidad de procesamiento, procesamiento auditivo, razonamiento cuantitativo y habilidades de lectura y escritura" (Pérez \& Medrano 2013, p. 107)

Gardner en 1983 propone las inteligencias múltiples: inteligencia lingüística, lógico matemático, naturalista y espacial. Este proceso nos lleva a definir la inteligencia como la capacidad que tienen las personas para resolver problemas, donde se manifiestan un conjunto de habilidades y capacidades para adaptase al entorno (Rigo \& Donolo 2013, Ardilla 2010, Anastasi 1998). 


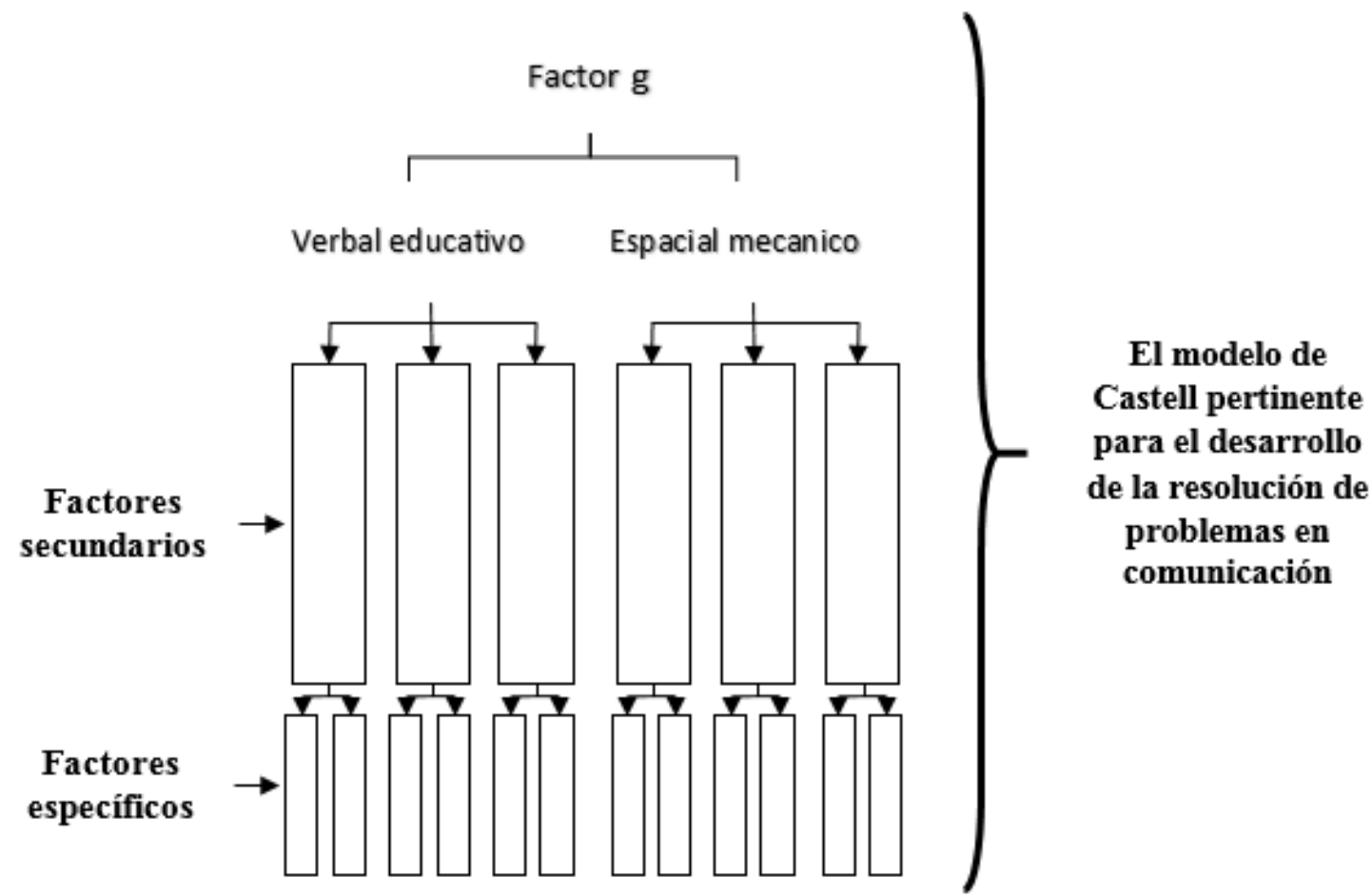

Figura 2. Modelo de la inteligencia fluida de Castell, 1967.

Martín y Boeck (2000) realizan estudios de la inteligencia emocional, que la definen como la comprensión de las emociones por las mismas personas que permite el mejoramiento de la calidad de vida.

\section{La inteligencia triárquica.}

Fue propuesta por Sternberg (1985), cuyo objetivo fue la comprensión de los procesos de la resolución de problemas. Kholer (2008) realizó el análisis psicométrico del instrumento propuesto por Sternberg, Sternberg Triarchic Abilities Test (STAT), cuyos resultados demostraron la confiabilidad y validez del instrumento, respondiendo al modelo centrado en el factor "G", que para algunos investigadores resultan insuficiente porque no explica el cómo se produce los procesos de la inteligencia. Más adelante, Gardner y Sternberg, el modelo multifactorial, como la inteligencia musical y la conciencia social entre otros.

En este marco, Sternberg (1985) plantea la "Teoría Triárquica de la Inteligencia de Robert Sternberg", que está centrada en los procesos cognitivos, donde realiza la codificación, almacenamiento y combinación de la información, para resolver problemas o dar respuestas a situaciones complejas. De allí, que plantea que el logro de las competencias cognitivas el ser humano requiere tener equilibrio de la inteligencia en sus tres componentes: "habilidad analítica, práctica y creativa".

Para las habilidades analíticas, se requiere realizar los procesos de planificar, ejecutar y evaluar los procesos mentales; la habilidad práctica, desarrolla los procesos de codificar, seleccionar y automatizar dichos procesos mentales; la habilidad práctica, donde se desarrollan los procesos de creatividad, donde se aplican las ideas en la realidad, es decir, la resolución de problemas. 


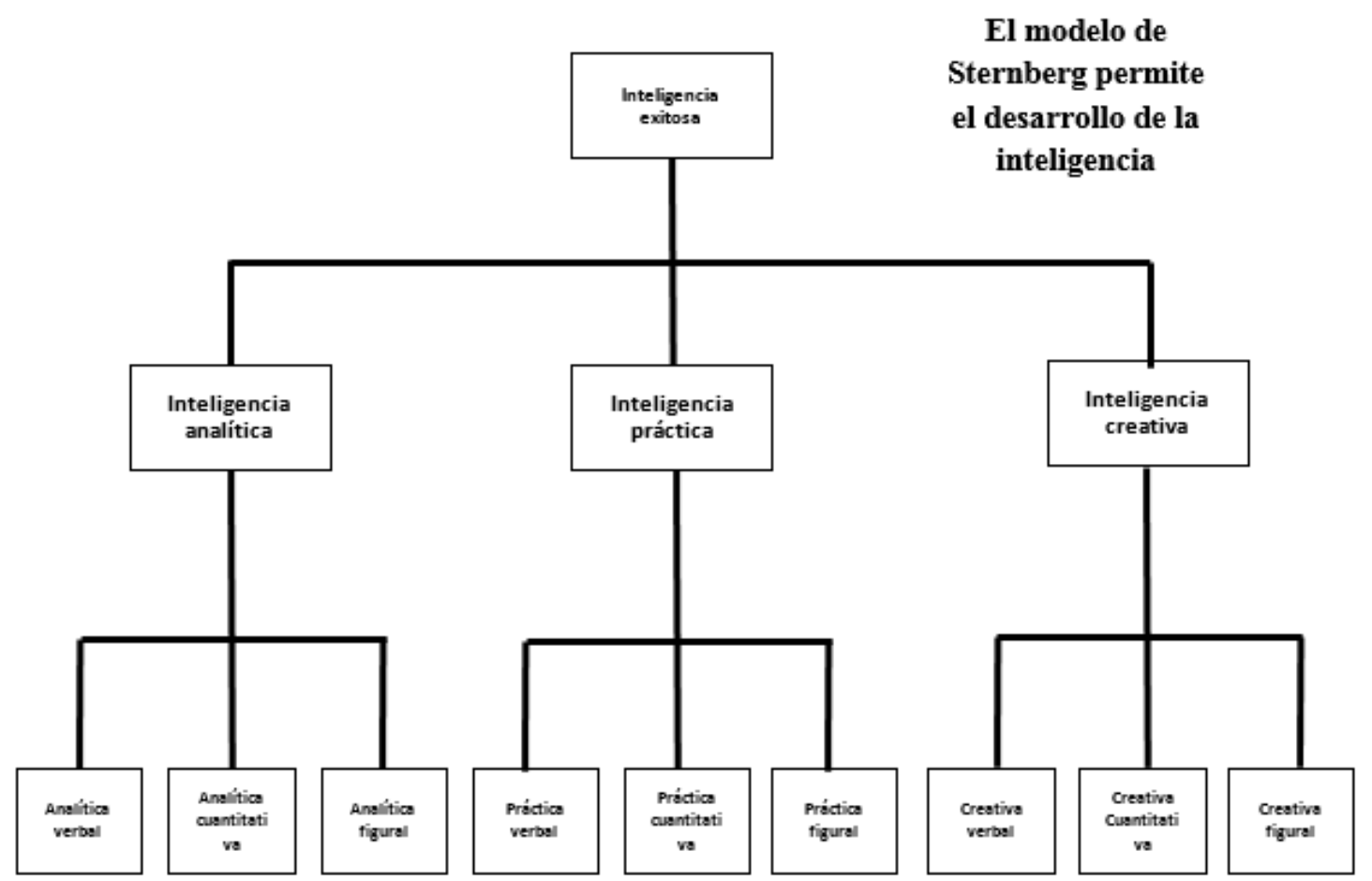

Figura 3. La inteligencia exitosa en sus tres factores, propuesto por Sternberg.

\section{Método}

La investigación se realizó desde dos perspectivas, aunque ejecutadas simultáneamente. La primera perspectiva fue realizada desde el enfoque cuantitativo, a través de una investigación aplicada, que también la denominan "empírica" donde se aplica un conocimiento a una realidad y cuyo resultado genera la validación a ampliación de ese conocimiento, para nuestro caso el uso de la técnica de mapas mentales en el desarrollo de la inteligencia triárquica. (Sánchez \& Reyes 2015; Vara 2015; Hernández, Fernández \& Baptista 2014; Bernal, 2011). Asimismo, se utilizó como diseño el cuasi experimental, con pruebas de pretest y post test, para el grupo experimental y control; este tipo de diseño permite la comparación entre ambos grupos, donde al inicio el control presenta semejanzas al experimental, permitiendo determinar si existen diferencias como producto de aplicación de algún programa. (White \& Sabarwal, 2014). La población estuvo conformada por cuarenta estudiantes de una institución educativa del nivel de secundaria del Cercado de Lima, donde tanto el grupo experimental como el control fue constituido por veinte estudiantes. Se utilizó el instrumento de Sternberg Triarchic Abilites Test (STAT), validado por Kholer (2008), y que tuvo una confiabilidad de KR de 0.810, estableciendo muy confiable para su aplicación. Se utilizaron estadísticas que demostraron al final del proceso existe diferencia de grupos entre el experimental y control, así como del pretest como del post test.

Asimismo, en forma simultánea se empleó la investigación cualitativa, a través de estudio de caso, que de acuerdo a Simons (2011) se caracteriza por ser un estudio exhaustivo, donde es evaluado el fenómeno a través de diversas perspectivas, pero en contexto de desarrollo, para tener la comprensión holística del fenómeno a estudiar. "Yin 1994...señala cinco categorías de estudio de caso: explicativo, descriptivo, ilustrativo, exploratorio y evaluativo" (Simons, 2011, p. 43) Para nuestro caso se utilizó el explicativo, donde establecen las causas que originan los fenómenos.

Durante los procedimientos se emplearon un conjunto de sesiones de aprendizaje para el grupo experimental, donde se plasmó la técnica de mapas mentales, desde el proceso de enseñanza para su elaboración, manual como digital, que permitieron diseñar un conjunto de estrategias para desarrollar y fortalecer la inteligencia exitosa de los estudiantes. Asimismo, durante la ejecución de las sesiones se emplearon diversas técnicas cualitativas, como la observación, entrevistas con 
los estudiantes participantes, análisis documental tanto de la planificación de las sesiones, instrumentos de evaluación, así como de los resultados formativos.

\section{Resultados}

Los primeros resultados estadísticos obtenidos en el pre test y post test se puede observar los diversos niveles de la inteligencia exitosa en sus tres dimensiones: así en la inteligencia analítica existe un avance significativo en el $10 \%$ de los estudiantes del nivel bajo a medio; en la inteligencia creativa se observa el desplazamiento del $10 \%$ del nivel bajo al nivel medio y alto; en la inteligencia práctica se observa el desplazamiento del nivel bajo al nivel medio del 3,3\% de los estudios. Estos resultados evidencian el mejoramiento de las habilidades analítica, creativa y práctica en los estudiantes debido a la aplicación de los mapas mentales.

Tabla 2.

La inteligencia exitosa -pretest post test- en los estudiantes del grupo experimental.

\begin{tabular}{lcccccc}
\hline & \multicolumn{2}{c}{$\begin{array}{c}\text { Desarrollo de la } \\
\text { habilidad analítica }\end{array}$} & \multicolumn{2}{c}{$\begin{array}{c}\text { Desarrollo de la } \\
\text { habilidad creativa }\end{array}$} & \multicolumn{2}{c}{$\begin{array}{c}\text { Desarrollo de la habilidad } \\
\text { práctica }\end{array}$} \\
\cline { 2 - 7 } & Pretest & Post test & Pretest & Post test & Pretest & Post test \\
\hline Bajo & $\%$ & $\%$ & $\%$ & $\%$ & $\%$ & $\%$ \\
Medio & $13.3 \%$ & $3.3 \%$ & $20.0 \%$ & $6.7 \%$ & $3.3 \%$ & $0.0 \%$ \\
Alto & $76.7 \%$ & $86.7 \%$ & $70.0 \%$ & $80.0 \%$ & $86.7 \%$ & $90.0 \%$ \\
Total & $10.0 \%$ & $10.0 \%$ & $10.0 \%$ & $13.3 \%$ & $10.0 \%$ & $10.0 \%$ \\
& $100.0 \%$ & $100.0 \%$ & $100.0 \%$ & $100.0 \%$ & $100.0 \%$ & $100.0 \%$
\end{tabular}

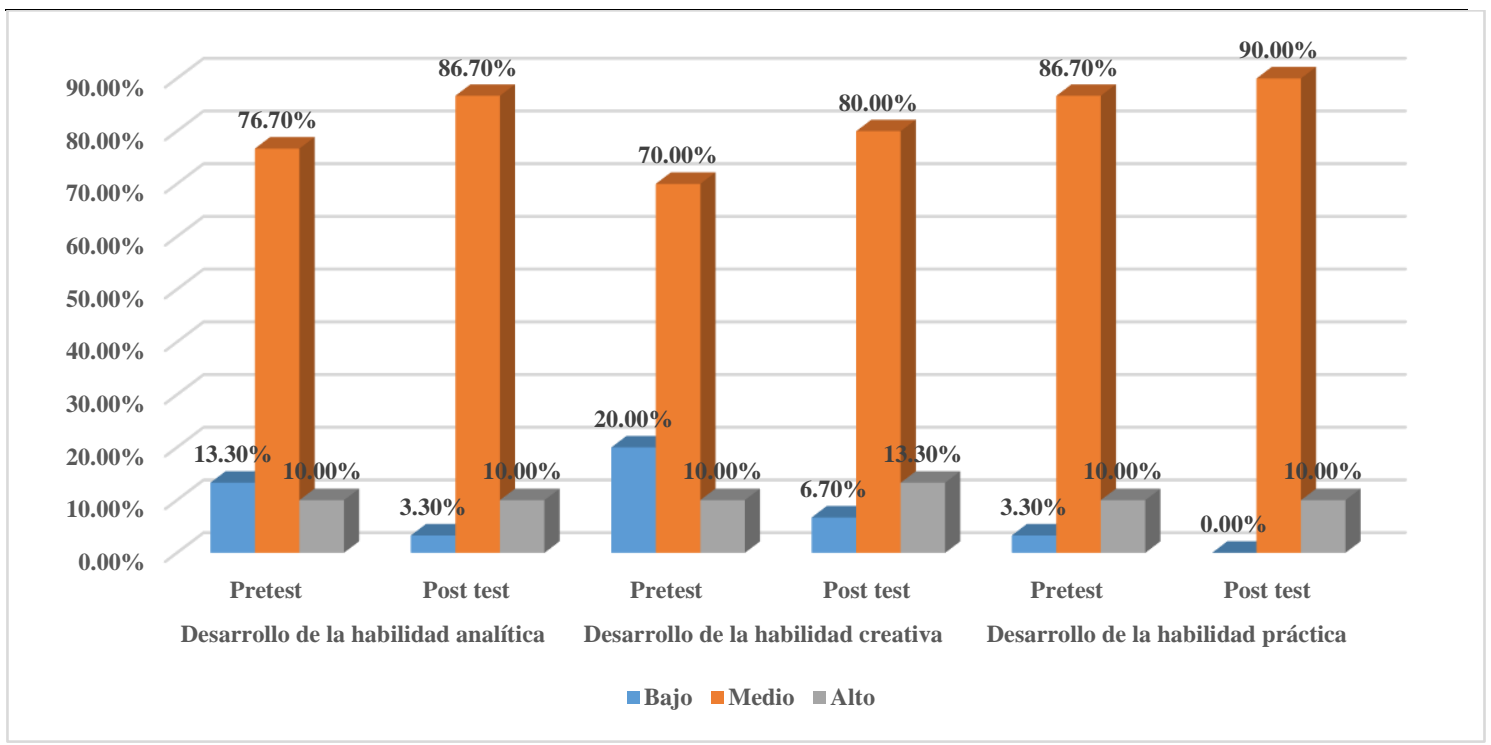

Figura 4. La inteligencia exitosa -pretest post test- en los estudiantes del grupo experimental.

Asimismo, los estadísticos de prueba utilizados para ver la diferencia de grupos $-\mathrm{U}$ de Mann Whitney- nos demuestran que existen diferencias estadísticas significativas entre el grupo control y experimental, en las tres habilidades de la inteligencia exitosa, donde la habilidad analítica presenta la significatividad bilateral de .000 y un valor de $\mathrm{T}$ tabla de -4.004 donde expresa que el experimental ha mejorado por acción de los mapas mentales; lo mismo se expresa en la habilidad creativa - .000 y -5.144 y habilidad práctica - .000 y -4.397. 
Tabla 3.

La inteligencia exitosa estadísticos de prueba en los estudiantes del grupo control-experimental

\begin{tabular}{|c|c|c|c|c|c|c|}
\hline & \multicolumn{2}{|c|}{ Inteligencia analítica } & \multicolumn{2}{|c|}{ Inteligencia creativa } & \multicolumn{2}{|c|}{ Inteligencia práctica } \\
\hline $\begin{array}{l}\text { Estadísticos } \\
\text { de prueba }\end{array}$ & $\begin{array}{c}\text { Pre test } \\
\text { control - } \\
\text { experimental }\end{array}$ & $\begin{array}{l}\text { Post test } \\
\text { control - } \\
\text { experimental }\end{array}$ & $\begin{array}{c}\text { Pre test } \\
\text { control - } \\
\text { experiment } \\
\text { al }\end{array}$ & $\begin{array}{c}\text { Post test } \\
\text { control - } \\
\text { experiment } \\
\text { al }\end{array}$ & $\begin{array}{c}\text { Pre test } \\
\text { control - } \\
\text { experiment } \\
\text { al }\end{array}$ & $\begin{array}{l}\text { Post test } \\
\text { control - } \\
\text { experiment } \\
\text { al }\end{array}$ \\
\hline $\begin{array}{l}\text { U de Mann- } \\
\text { Whitney }\end{array}$ & 361.000 & 182.500 & 296.500 & 108.500 & 321.000 & 154.500 \\
\hline $\begin{array}{l}\text { W de } \\
\text { Wilcoxon }\end{array}$ & 826.000 & 647.500 & 761.500 & 573.500 & 786.000 & 619.500 \\
\hline $\mathrm{Z}$ & -1.344 & -4.004 & -2.366 & -5.144 & -1.945 & -4.397 \\
\hline $\begin{array}{l}\text { Sig. } \\
\text { asintótica } \\
\text { (bilateral) }\end{array}$ & .179 & .000 & .018 & .000 & .052 & .000 \\
\hline
\end{tabular}

Los datos estadísticos aplicados nos muestran el avance significativo de los estudiantes en relación a las habilidades de la inteligencia exitosa, en donde con los resultados del pretest se establece una línea de base. Se realizó la explicación a ambos grupos en forma separada con la presencia del investigador. Al inicio se explicó la finalidad de la investigación a los estudiantes de ambos grupos, mostrando en la mayoría de ellos entusiasmo y deseo de participación. El docente, de manera sencilla se detallaba los pasos y procedimientos que se realizarían durante las sesiones de aprendizaje, mostrando que ha realizado la planificación adecuada de las sesiones de aprendizaje.

La evaluación de dichas sesiones se pudo observar que estaban conformadas por los siguientes aspectos: título de la sesión, competencias, capacidades e indicadores a desarrollar, secuencia didáctica, cierre, materiales y recursos en donde se destaca el "storyboarding", que es un conjunto de figuras que detallan los procedimientos seguir. Todas ellas denotaban coherencia en su construcción, destacándose la estrategia metodológica a seguir. La planificación requiere en los contextos actuales mirar hacia el futuro para una toma de decisiones coherentes, estrategias que permitan lograr los objetivos propuestos (Aguerrondo, 2014).

En la primera sesión de aprendizaje del grupo experimental, se han utilizado diversas técnicas de lectura, enfatizando el uso de subrayado, cuadros sinópticos para la localización de aspectos relevantes, permitiendo determinar las ideas principales y secundarias del texto. Paso seguido, se dio el aprendizaje en el uso y elaboración del mapa mental, donde los estudiantes han comenzado a delinear lo relevante de la lectura en esquema que desarrollan. Es importante destacar el uso de colores e iconos en su elaboración.

Los estudiantes al inicio se muestran entusiasmados, pero en el proceso de la sesión muestran dificultades en su aprendizaje. Ellos manifestaron al final de la primera sesión, que les parecía atractivo los mapas mentales y que les permitían participar activamente en la clase, pero que todavía no comprendían como articular la lectura con el organizador. El docente explicó que era la fase inicial y que por ser utilizada la técnica apropiadamente, todavía existían algunos vacíos que cubrir. Asimismo, recién están los inicios de como relacionar las habilidades analíticas del mapa. Por ello Simons (2011) explicaba que en esta fase es fundamental la negociación en base a la emisión de las opiniones de las participantes, dándoles respuestas pertinentes y adecuadas que permitan mantener la motivación y participación activa del estudiante.

En la entrevista a profundidad realizada a la experta en temas didácticos Dra. Sánchez, explicaba que una buena planificación implica para el docente cumplir con una serie de procedimientos sean heurísticos o algorítmicos, que le van a permitir el logro de los objetivos didácticos planteados en la sesión. Si bien el esfuerzo del docente en cumplir los procedimientos 
indicados en la sesión, encontraba alguna resistencia en los estudiantes. El diálogo con ellos, indicaron que no entendían algunos procedimientos realizados en clase.

De la segunda a la cuarta sesión de aprendizaje, el docente ha mejorado su comunicación con los estudiantes y éstos a su vez comprenden mejor el proceso de construcción de los aprendizajes. Esto denota asimismo el proceso de desarrollo de las habilidades de la inteligencia triárquica. En la habilidad analítica, los estudiantes comienzan a concebir el mapa mental de manera holística, la lectura como un todo y de partes articuladas lógicamente. Y esto expresado en cada bifurcación e ícono realizado. Esto también como desarrollo de la creatividad, expresada en el uso de colores, iconografías, dándole organización y coherencia a la información analizada. Por último, vienen comprendiendo la utilidad de este trabajo, porque visualizan la sistematización de la lectura analizada y los resultados que vienen obteniendo en la evaluación formativa.

Tres procesos vienen ocurriendo simultáneamente, el mejoramiento de la aplicación de la técnica en la elaboración de los mapas mentales, el desarrollo de las competencias analíticas y el desarrollo de las habilidades triárquica. Indudablemente la experiencia nos demuestra que las variables indicadas están íntimamente relacionadas, dándole coherencia y validez interna a la investigación, a través de la descripción profunda del proceso.

Para la Dra. Menacho, experta psicopedagoga explica que para el desarrollo de la inteligencia en los estudiantes es fundamental los estímulos tanto académicos como motivacionales; por ejemplo -afirma- que las tareas son atractivas, rompiendo los temores propios en su realización, la hacen ellos mismos, donde en realidad expresan su alegría al lograr la meta esperada y esto en conjunto es su motivación y motor de seguir aprendiendo.

En las sesiones a partir de la quinta, el proceso evolutivo de su aprendizaje se desarrolló sin alcanzar las metas esperadas. Sin embargo lo logrado nos demuestra que los mapas mentales son herramientas claves para el desarrollo de la inteligencia triárquica, donde los resultados indicaron que en grupo reducido no logro avanzar en el desarrollo de la inteligencia y que un grupo mayoritario se ubicó en un nivel intermedio.

Los expertos consultados, tanto la Dra. Sánchez como la Dra. Menacho coinciden que este proceso que requiere de tiempo considerable y que sería magnífico si se inicia desde los primeros grados en la escuela, permitiendo con ello no solo de las competencias sino también de la inteligencia

Durante la ejecución de los procesos de aprendizaje se observó que el docente utilizó estrategias metodológicas activas, donde la interacción con los estudiantes es permanente, estimulando permanentemente su actuar, realizando conjuntamente con ellos el análisis de las lecturas escogidas y el uso de los mapas mentales para el propósito de las clases.

\section{Discusión}

Los mapas mentales como estrategia en el desarrollo de la inteligencia en estudiantes de secundaria nos muestran que son excelentes herramientas didácticas. González et al (2015) llega a la conclusión de que son una técnica efectiva para desarrollar al máximo las relaciones interpersonales, pero son más que eso, son en sí mismos, elementos de crecimiento de la inteligencia exitosa.

Martínez (2017) por su parte, llega a establecer una alta influencia entre los mapas mentales y el pensamiento creativo. Sin embargo, el presente artículo extiende esta aseveración al ámbito de procesos de inteligencia, que son más y que no niegan, sino incluyen entre otros al raciocinio de tipo creativo. Concordando con Kholer (2008), se puede añadir que las estrategias de aprendizaje permiten el desarrollo de la inteligencia triárquica. Asimismo, las evidencias 
cuantitativas y cualitativas nos llevan a afirmar que el uso pertinente y adecuado de las estrategias ha permitido el desarrollo de la inteligencia exitosa de manera global.

Para Molina y Martinez (2016), los mapas mentales constituyen una estrategia didáctica muy efectiva que logra el ansiado aprendizaje significativo. Requisito indispensable para conseguir el ansiado aprendizaje significativo. Sin embargo; solo con la consolidación de la inteligência exitosa, se puede aspirar al avance integral (Kholer, 2008b; Rigo \& Donolo, 2013).

Y por último, el factor tiempo puede ser un factor a considerar en otras investigaciones, donde se planifiquen un número mayor de sesiones de aprendizajes, incluyendo mayores estrategias de comprensión lectora alineada al uso de los mapas mentales; asimismo, incluir mayor número de horas en el uso de software, de manera que tengan herramientas para el complemento del mapa mental y si es aplicado a grupos de estudiantes, escoger un batería de lecturas, de acuerdo al grado y edad, que posibiliten la obtención de los resultados previstos.

\section{Referencias}

Aguerrondo, I. (2014). Planificación educativa y complejidad: gestión de las reformas educativas. Cadernos de Pesquisa, 44(153), 548-578. Doi: https://doi.org/10.1590/198053142910

Ardila, A., \& Ostrosky-Solís, F. (1991). Diagnóstico del daño cerebral: enfoque neuropsicológico. México D.F: Trillas.

Ardilla, R. (2010). Inteligencia. ¿Qué sabemos y qué nos falta por investigar? Revista de la Academia Colombiana de Ciencias Exactas, Físicas y Naturales, 35(134), 97-103. Recuperado de: http://www.scielo.org.co/pdf/racefn/v35n134/v35n134a09.pdf

Bernal, C. (2011) Metodología de la investigación. Administración, economía, humanidades y ciencias sociales (3ra ed.). México. Prentice Hall.

Blanco Púa, Y. A., \& Hoz Montero, O. E. D. L. (2017). Mapas mentales como estrategia para el fortalecimiento de la conceptualización de los sistemas de ecuaciones lineales $2 X 2$. (Tesis de Maestría), Universidad del Norte, Barranquilla.

Buzan, T. (1996). El libro de los mapas mentales. Barcelona: Editorial Urano.

Gardner, H. (1983). Frames of mind: The theory of multiples intelligences. New York: Basic.

Gonzáles Moreyra, R. (1998). Nivel de comprensión lectora en los universitarios iniciales. (Tesis de Maestría), Universidad Nacional Mayor de San Marcos, Lima.

González, J. M. M., Requena, B. E. S., \& Díaz, V. M. (2015). Los mapas mentales, una técnica para potenciar las relaciones interpersonales. Tendencias Pedagógicas, 24, 401-414. Recuperado de: https://revistas.uam.es/tendenciaspedagogicas/article/view/2114

González, J. M. M., Pareja, E. F. H., \& Gea, E. M. V. (2016). Opiniones de estudiantes universitarios acerca de la utilización de mapas mentales en dinámicas de aprendizaje cooperativo. Perfiles Educativos, 38(153), 136-151. Recuperado de: http://www.iisue.unam.mx/perfiles/articulos/2016/n153a2016/mx.peredu.2016.n153.p136 -151.pdf

Gordillo Al., \& Flórez, M. del P. (2017). Los niveles de comprensión lectora: hacia una enunciación investigativa y reflexiva para mejorar la comprensión lectora en estudiantes universitarios. Actualidades Pedagógicas, (53), 95-107. Recuperado de: http://revistas.lasalle.edu.co/index.php/ap/article/view/1048

Hernández, R., Fernández, C., \& Baptista, P. (2014), Metodología de la Investigación. (6ta. Ed.). México: Mc Graw Hill Education.

Kohler, J. (2008a). Relación entre habilidades intelectuales, estrategias de aprendizaje y rendimiento académico en estudiantes de psicología del $1^{\circ}$ al $4^{\circ}$ ciclo de una universidad particular (Tesis de Maestría), Universidad de San Martín de Porres, Lima.

Kholer, J. (2008b) Análisis Psicométrico de la Prueba de Habilidades Triárquicas de Sternberg, Revista Iberoamericana de Diagnóstico y Evaluación - e Avaliação Psicológica, 2(26) 167-191. Recuperado de: https://www.redalyc.org/html/4596/459647347009/

Martín, D., \& Boeck, K. (2000) Qué es Inteligencia Emocional. Madrid, España, EDAF. 
Martínez, G. A. R. (2017). La cartografía mental y su incidencia en el pensamiento creativo. Revista $\quad$ Q, 5(10), 1-23. Recuperado de: https://revistas.upb.edu.co/index.php/revista_Q/article/view/7796

Molina, L., \& Martinez, M. B. (2016, October). El mapa mental: una estrategia didáctica que genera aprendizaje significativo. En, 3er Simposio Internacional y 4to Colóquio Regional de Investigación Educativa y Pedagógica.

Montero, E. G., \& De la Morena Taboada, M. (2015). Analizando el autoconcepto y la imagen: aplicación del mapa mental a la construcción de la marca personal. Opción: Revista de Ciencias Humanas y Sociales, (2), 396-424. Recuperado de: http://www.produccioncientifica.luz.edu.ve/index.php/opcion/article/view/20398

Ontoria, A., Gómez, J. P., Molina, A. \& Luque de, A. (2006). Aprender con Mapas Mentales. Madrid: Narcea.

Pérez, E; Medrano, L. (2013). Teorías contemporáneas de la inteligencia. Una revisión crítica de la literatura. Psiencia. Revista Latinoamericana de Ciencia Psicológica, 5(2), 105-118. Doi: http://dx.doi.org/10.5872/psiencia.v5i2.113

Pizarro Cherre, F. (2008). Aplicación de los mapas mentales en la producción de textos en estudiantes del ciclo I de instituciones de educación superior. (Tesis de maestría). Universidad Nacional Mayor de San Marcos, Lima.

Rigo, D. y Donolo, D. (2013). Tres enfoques sobre inteligencia: un estudio con trabajadores manuales. Estudios de Psicología, 30(1), 39-48. Recuperado de: http://ri.conicet.gov.ar/handle/11336/9766

Simons; H. (2011). El estudio de caso: teoría y práctica. Madrid: Ediciones Morata.:

Sternberg, R. (1985). Más allá del cociente intelectual: una teoría triárquica de la inteligencia humana. Archivo CUP.

Sternberg, R. (1985). The triarchic mind. New York: Penguin Books.

Sternberg, R. (1987). Inteligencia humana I. La naturaleza de la inteligencia y su medición. Barcelona: Paidós.

Sternberg, R. J. (2006). The Rainbow Project: mejorando el SAT a través de evaluaciones de habilidades analíticas, prácticas y creativas. Inteligencia, 34, 321 - 350. Doi: https://doi.org/10.1016/j.intell.2006.01.002

Sánchez, H., \& Reyes, C. (2015), Metodología y Diseños en la Investigación Científica (5ta Ed.). Lima: Business Support Aneth SRL

Smith, F. (2005). Para darle sentido a la lectura-aprendizaje. Madrid: Visor.

Vara, A. (2015). 7 pasos para elaborar una tesis. Lima: Macro EIRL.

White, H., \& Sabarwal; S. (2014). Diseño y métodos cuasiexperimentales. Síntesis metodológicas: evaluación de impacto $n^{o}$ 8. Florida: Centro de Investigaciones de UNICEF.|2|| 